\title{
Bedding Material
}

National Cancer Institute

\section{Source}

National Cancer Institute. Bedding Material. NCI Thesaurus. Code C90366.

That which comprises the place where a subject sleeps. 